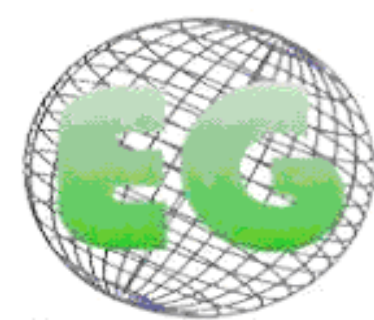

ISSN 1695-6141 $N^{\circ} 22$
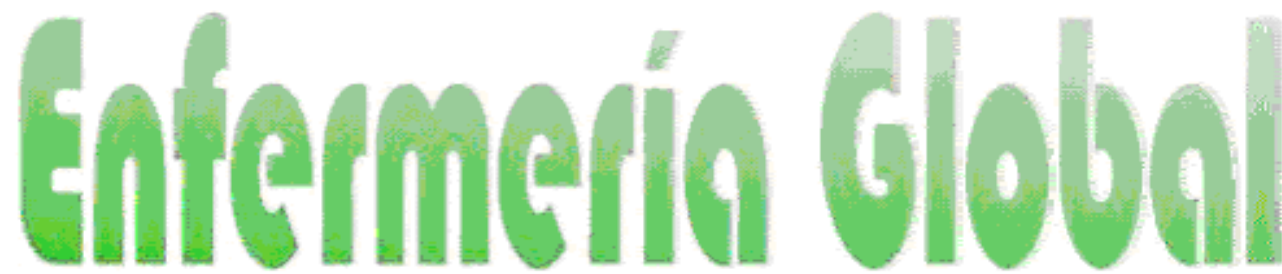

Revista electrónica trimestral de Enfermería

Abril 2011

www.um.es/egloball

REVISIONES

\title{
REVISIÓN SOBRE LA INTERACCIÓN ENTRE FÁRMACOS Y NUTRICIÓN ENTERAL
}

\author{
REVISÃO SOBRE A INTERAÇÃO ENTRE FÁRMACOS E NUTRIÇÃO \\ ENTERAL
}

*Schutz, V., "*Dopico da Silva, L., " ${ }^{* * *}$ Praça, BFM., "**Dos Santos, MER.

*Profesora Doctora. **Profesora Doctora, Coordinadora del Curso de Pós-Graduação Lato-Sensus Enfermagem Intensivista. *** Enfermeras del Curso de Pós-Graduação Latu-Sensus Enfermagem Intensivista.Universidad del Estado de Rio de Janeiro (UERJ). Brasil.

\begin{abstract}
Palabras clave: Medicamentos; Nutrición enteral; Enfermería.
Palabras chave: Medicamentos; Nutrição enteral; Enfermagem

Keywords: Drug; Enteral nutrition; Nursing.
\end{abstract}

\section{RESUMEN}

Este artículo se refiere a la administración de fármacos concomitante a la administración de dieta por sonda.

Objetivo: Buscar las evidencias disponibles en la literatura que aborden el tema interacción entre las fármacos y nutrición enteral administrados por sonda.

Metodología: Revisión que usó datos de bases electrónicas.

Resultados: Encontrados 62 artículos y seleccionados siete.

Discusión: Las clases farmacológicas encontradas fueron los antiepiléticos (43\%), antibióticos (43\%) y anticoagulantes (14\%). La clase que demostró mayor riesgo de interacción con la nutrición enteral fueron los antiepiléticos, principalmente la fenitoína. Los antibióticos no demostraron significativas reducciones en términos de niveles séricos y la Warfarina, único anticoagulante mencionado, también presentó riesgo de interacción con la nutrición enteral.

Conclusión: Es necesario desarrollar iniciativas como el hábito de lavar la sonda, interrumpir la dieta enteral, hacer exámenes de laboratorio para prevenir posibles interacciones entre fármacos y la nutrición. 


\section{RESUMO}

Artigo sobre a administração de fármacos concomitante à administração de dieta por sonda.

Objetivo: buscar as evidências disponíveis na literatura que abordem o tema interação entre fármacos e nutrição enteral administrados por sondas.

Metodologia: revisão que usou dados de bases eletrônicas.

Resultados: foram encontrados 62 artigos e selecionados sete.

Discussão: as classes farmacológicas encontradas foram a dos antiepiléticos (43\%), antibióticos $(43 \%)$ e anticoagulantes (14\%). A classe que mais demonstrou risco de interação com a nutrição enteral foi a dos antiepiléticos, principalmente, com a fenitoína. Os antibióticos não demonstraram significativos decréscimos em termos de níveis séricos e a Warfarina, único anticoagulante mencionado, também apresentou risco de interação com a nutrição enteral.

Conclusão: torna-se necessário desenvolver iniciativas como o hábito de lavar a sonda, interromper a dieta enteral, monitorar exames laboratoriais para prevenir possíveis interações entre fármacos e a nutrição enteral."

\section{ABSTRACT}

Article on the drug administration of concomitant enteral nutrition. Objective: To find the available evidence in the literature that addresses the issue of drug interaction and enteral nutrition administered by tube. Methodology: review that used data from electronic databases. Results: 62 articles were found and seven were selected. Discussion: The classes of drug were antiepileptics $(43 \%)$, antibiotics (43\%) and anticoagulants (14\%). The class that has most risk of interaction with enteral nutrition was that of antiepileptics, especially with phenytoin. Antibiotics showed no significant decreases in serum and warfarin, the only anticoagulant mentioned, also presented a risk of interaction with enteral nutrition. Conclusion: it is necessary to develop initiatives such as the habit of washing the probe, stopping the enteral feeding, monitoring laboratory tests to prevent possible interactions between drugs and enteral nutrition

\section{INTRODUCCIÓN}

En la Unidad de Terapia Intensiva (UTI) se impone la necesidad de inversiones en recursos humanos, tecnológicos y farmacológicos, los cuales representan insumos indispensables para la reducción de morbimortalidad de los pacientes. En la práctica, la combinación de medicamentos (producto farmacéutico técnicamente obtenido, o elaborado, con finalidad profiláctica, curativa, paliativa o diagnóstica) ${ }^{(1)}$ con diferentes efectos es una estrategia útil para potenciar los efectos terapéuticos de los agentes combinados en relación al uso aislado del fármaco (substancia activa que es definida como el principio activo del medicamento).

En relación a la terapia medicamentosa es importante alcanzar la mejor respuesta clínica posible en términos de eficacia y seguridad para el paciente, y esto no depende solo del diagnóstico correcto y de la prescripción del tratamiento farmacológico adecuado. La evaluación de todos los factores que pueden modificar la respuesta farmacológica esperada exige conocimiento de las fuentes de variabilidad, para que las interacciones entre medicamentos y nutrientes sean identificadas.

Ya el soporte nutricional es parte vital de la terapia en la mayoría de los pacientes en la UTI, teniendo beneficios reconocidos como la diminución de la morbimortalidad, disminución del 
tiempo de hospitalización, caída en la tasa de infección y una mejora en la inmunidad de estos pacientes $^{(2)}$.

La nutrición enteral, siempre que sea posible, debe ser la primer opción de terapia nutricional, pues tiene muchas ventajas en relación a la nutrición parenteral, como menor posibilidad de desplazamiento bacteriano, ser más fisiológica y barata, menor probabilidad de contaminación, composición variable para cada paciente y ser formulada a partir del estado clínico, nutricional y de la necesidad de reposición protéico-calórica de cada paciente $^{(2)}$.

Resulta común cuidar de un gran número de pacientes que reciben por sondas, nutrición enteral y medicamentos y estos pueden interactuar con la nutrición, lo que puede alterar los niveles séricos del medicamento, su absorción o incluso obstruir la sonda ${ }^{(3)}$. Una interacción fármaco-nutriente es definida como :"alteracciones producidas en los efectos terapéuticos de un medicamento en razón de la ingestión concomitante con alimento ". ${ }^{(2: 34)}$ Un ejemplo de interacción es la fenitoína, ya que los alimentos pueden reducir su biodisponibilidad (grado en que una droga u otra sustancia llega a la circulación general y se pone a disposición del órgano o tejido) - tornando necesaria la alteración del horario de administración o ajuste de dosis, para evitar el uso de dosis subterapeuticas.

Esta investigación estudia la interacción entre fármacos y nutrición enteral cuando el equipo de enfermería administra ambas por sondas usadas para la nutrición enteral. ${ }^{(1)}$ El objetivo fue describir la producción científica acerca de interacción fármaco-nutrición enteral a partir de los planteamientos en relación a los medicamentos encontrados en la producción rastreada. Entendiendo que es el enfermero que realiza o supervisa la administración de medicamentos y nutrición enteral quien debe conocer los fármacos cuya velocidad de absorción y/ o la cantidad absorbida, pueden verse afectadas en presencia de alimentos (4-5). Conocer los aspectos involucrados en interacción fármaco-nutriente garantizará mejores resultados a los pacientes, Desde una perspectiva ampliada de evaluación y control de resultados acerca del cuidado de enfermería a pacientes que reciben medicamentos y nutrición enteral, esta investigación puede ayudar a evitar aspectos que comprometan los resultados de la terapia medicamentosa. También puede contribuir al desarrollo de protocolos para grupos específicos de medicamentos, lo que beneficiará el cuidado a los enfermos.

\section{METODOLOGÍA}

Se trata de revisión bibliográfica con la seguiente pregunta planteadora: ¿Cual es la producción científica acerca de la interacción fármaco-nutriente en pacientes adultos?

La identificación del objeto ocurrió en las bases MedLine, Lilacs, Scielo, ScienceDirect assi también la base de datos secundaria OVID, cumpliendo la recomendación de que la encuesta provenga de fuentes diversas. (6) Los criterios de selección fueron artículos publicados en portugués, inglés y español, con resúmenes disponibles en las bases de datos citadas; indexados por medicamentos/fármacos; nutriçao enteral / enteral nutrition/nutrición enteral; publicados entre 1997 a 2007, sobre pacientes adultos (18 años o más); investigaciones de campo; citar la administración de fármacos por sonda y la interacción fármaco-nutriente. Las estrategias usadas para encontrar los artículos fueron adaptadas para cada base de datos, de acuerdo con la especificidad de cada base, siendo guiadas por la pregunta y los criterios de selección. La encuesta se realizó entre marzo y julio de 2008. Se encontraron 62 publicaciones y se seleccionaron siete. Los resultados se agruparon en categorías definidas a partir de los fármacos presentes en la muestra. 


\section{RESULTADOS}

Los artículos tuvieron como autores, médicos, farmacéuticos y nutricionistas. Las publicaciones fueron agrupadas en categorías de antiepiléticos ${ }^{(7-9)}$, de antibióticos ${ }^{(10-12)}$ y anticoagulantes $^{(13)}$. Solamente cuatro estudios fueron concluyentes para interacción siendo tres estudios de la clase de antiepiléticos (fenitoína) y uno relacionado con anticoagulantes (warfarina)

\section{Categoria de los antiepiléticos}

Un estudio investigó el nivel sérico de la fenitoína en 22 pacientes hospitalizados que hacían uso de este medicamento administrado por sonda al mismo tiempo que la nutrición enteral. Estos participantes se dividieron en dos grupos. En un grupo, la nutrición enteral fue interrumpida durante una hora después de la dosis de fenitoína y en el otro no hubo esta interrupción. La investigación concluyó que una hora de descanso para la nutrición enteral tras la dosis de fenitoína, aumenta los niveles séricos de fenitoína en casi $90 \%$, y ayuda a mantener el nivel terapéutico del mismo ${ }^{(7)}$

Otro estudio ${ }^{(8)}$ se llevó a cabo con 25 pacientes ingresados en un hospital que recibían nutrición enteral por sonda. Se observaron 123 medicamentos administrados junto con la nutrición enteral siendo necesarias varias intervenciones farmacéuticas, en nueve casos para cambiar la fórmula farmacéutica sólida por líquida. Otras cuatro intervenciones ocurrieron para cambiar fórmulas sólidas retard o entéricas; en otros dos casos se orientó acerca de la administración del fármaco distante en el tiempo de la dieta y por último debido a interacción entre almagato y ciprofloxacino, se recomendó no mezclarlos durante la preparación y su administración por sondas.

Otra investigación ${ }^{(9)}$ hecha con 440 pacientes internados en un hospital, recibiendo medicación y nutrición enteral por sondas, se identificaron 33 casos de interacción fármaconutrientes. En once de estos casos, el fármaco mencionado por el estudio fue la fenitoína. El estudio concluyó que existe interacción con la nutrición enteral cuando es administrado concomitante por sonda, teniendo sus niveles séricos disminuidos.

\section{Categoria de los antibióticos}

Una publicación ${ }^{(10)}$ acerca del efecto de la nutrición enteral en la farmacocinética del moxifloxacino se hizo con doce voluntarios jóvenes sanos que se dividieron en tres grupos con cuatro individuos cada uno. El grupo A recibió el fármaco por vía oral con agua; el grupo $\mathrm{B}$ lo recibió triturado con agua por la sonda; y el $\mathrm{C}$ recibió triturado por la sonda nasogástrica junto con la nutrición enteral. Se concluyó que la biodisponibilidad del moxifloxacino cuando se administra solo por sonda nasogástrica o junto con la nutrición enteral no es afectada, sin embargo la concentración sérica (cantidad de una substancia en relación al plasma sanguíneo ${ }^{12}$ ) máxima de moxifloxacino en el grupo $B$ disminuyó $5 \%$ y en el grupo $C$, en $12 \%{ }^{(9)}$

Un estudio(11) ${ }^{(1) a t o ́ ~ d e ~ l a ~ b i o d i s p o n i b i l i d a d ~ d e l ~ c i p r o f l o x a c i n o ~ a d m i n i s t r a d o, ~ i n i c i a l m e n t e ~ p o r ~}$ vía endovenosa y después por sonda nasogástrica en 12 pacientes críticos que también recebian nutrición enteral haciendo medidas seriadas de los niveles séricos del fármaco. Los resultados mostraron que la concentración sérica máxima del fármaco fue, aproximadamente, $44 \%$ inferior cuando administrado por sonda, pero su concentración sérica mínima fue similar en ambas rutas, concluyendo que es posible la continuidad de un 
tratamiento con ciprofloxacino vía sonda nasogástrica. El estudio sugiere unl aumento de la dosis en tres veces más que la dosis por vía endovenosa.

Autores $^{(12)}$ investigaron la biodisponibilidade del gatifloxacino (comercializado en Brasil con el nombre de Tequin) cuando administrado por vía endovenosa y por sonda enteral en dieciséis pacientes críticos que recibían nutrición por sonda. Estos sujetos fueron separados en dos grupos, donde un grupo recibía el fármaco por sonda con la interrupción de la nutrición enteral una hora antes y otra después de la administración del fármaco y otro grupo que recibía el remedio sin la interrupción de nutrición enteral. Se concluyó que la biodisponibilidad del gatifloxacino donde hubo interrupción de nutrición enteral fue de 98,5\% y $100 \%$ en el grupo donde la nutrición enteral no se interrumpió informando que no hubo interacción entre el antibiótico y la nutrición .

\section{Categoría de los anticoagulantes}

Una publicación ${ }^{(13)}$ aborda la administración concomitante de warfarina y nutrición por sonda en seis pacientes por lo menos por diez días consecutivos teniendo los mismos sus valores normales de Relação Normalizada Internacional (RNI). ). (EI RNI se basa en la relación del valor del tiempo de protrombina (TP) del paciente y la media de los valores normales de plasmas normales frescos, y representa la relación de TP si el test hubiese sido realizado con la tromboplastina IRP (International Reference Preparation). Esta padronización evita variaciones interlaboratoriales. El Tiempo de protrombina mide la vía extrínseca de la coagulación, prolongándose en las deficiencias selectivas o conjuntas de los factores II, V, VII y X. Como los cuatro factores son sintetizados en el hígado y tres de ellos son vitamina $\mathrm{K}$ dependientes (II, VII, X), el TP es utilizado más comúnmente en la monitorización de la terapia anticoagulante oral (warfarina).

Durante tres días consecutivos, la nutrición enteral fue interrumpida una hora antes y una hora después para administrar la warfarina y, en otro período también de tres días consecutivos, no hubo interrupción de nutrición enteral. Como resultado, en el período en que la nutrición no fue interrumpida para la administración del fármaco, el RNI disminuyó en $73 \%$ comparado con el período cuando fue interrumpido. Se concluye que hubo interacción entre warfarina y nutrición enteral.

\section{DISCUSIÓN DE LOS DATOS}

El soporte nutricional enteral en pacientes hospitalizados, puede ser administrado por sondas enterales que son clasificadas conforme a su topografia en gástricas (nasogástrica, orogástrica o gastrostomia), y pós-pilóricas (duodenales o yeyunales) ${ }^{(14)}$.

Los mecanismos responsables por interacciones fármaco-nutrientes suelen clasificarse en farmacocinéticos que son los que afectan a los procesos de liberación, absorción, distribuición, metabolismo y excreción (LADME) de medicamentos, y en farmacodinámicos los que provocan alteraciones en la respuesta farmacológica y/o efectos adversos de los fármacos, sin afectar su disposición en el organismo.

Autores $^{(5)}$ al tratar de interacción fármaco-nutrientes, afirman que aunque la mayoria de los estudios se centran en el efecto del fármaco sobre la nutrición enteral, lo más immportante sería el efecto de la nutrición enteral en la biodisponibilidad del medicamento y, como ejemplo, se cita la interacción de la fenitoína con la nutrición enteral. Tres estudios ${ }^{(7-9)}$ reportaron esta interacción, refiriendo el cuidado de detener la nutrición enteral por lo menos una hora antes y tras su administración. El mismo cuidado debe ser dispensado por 
enfermeros a la warfarina pues se ha demostrado relevancia clínica y estadística satisfactoria en la respuesta del RNI al interrumpirse la nutrición enteral antes y después de la administración de este fármaco ${ }^{(13)}$..

Respecto de la warfarina, se sabe que esta puede tener reducida la producción de cuatro factores de coagulación dependientes de la vitamina $\mathrm{K}$, o sea, significa que un paciente haciendo uso de terapia anticoagulante con warfarina, debe tener una ingesta de vitamina $\mathrm{K}$ adecuada para mantener el equilibrio de la anticoagulación.

Estudio $^{(11)}$ de ciprofloxacino reportó una disminución de $44 \%$ en el nível sérico de este medicamento al cambiar la vía de administración intravenosa para enteral por sonda, lo que justifica el cuidado del enfermero de interrumpir la nutrición enteral antes y después de la administración del fármaco.Estas interrupciones, dependiendo de la cantidad de medicamentos y el número de veces que se administran en unm paciente con nutrición que es continua o intermitente, puede afectar a la fecha de vencimiento de la dieta enteral y así reducir la ingesta de nutrientes al paciente. Para evitar el desperdicio de dieta y garantizar que el paciente reciba los nutrientes necesarios para su recuperación, el enfermero debe volver a calcular la velocidad de infusión de la dieta. Cuando el método de infusión es continuo, con la sonda en el estómago y peristaltismo normal, la velocidad de infusión puede ser aumentada gradualmente hasta la velocidad máxima de 100 a $125 \mathrm{ml} / \mathrm{h}$, una vez que el estómago tolera un mayor volumen de líquido en comparación con el intestino. Cuando la sonda se encuentra en el duodeno, se debe disminuir la velocidad de infusión a la mitad, ya que el duodeno tiene un cumplimiento menor. Para estos cambios hay que observar la tolerancia del paciente y su clínica. ${ }^{(13)}$

En la administración de medicamentos a través de una sonda gástrica, la enfermera debe recordar que la presentación de la misma es un factor a considerar, ya que determina su lugar de acción y su modo de preparación. Es decir, debe saber si la droga puede ser macerada o no, con lo que puede ser diluida y con qué componentes de nutrición enteral se puede interactuar.

Las presentaciones de medicamentos para su administración por vía enteral son cápsulas, tabletas, pastillas, elixires, emulsiones, suspensiones y jarabes. La administración de cápsulas y tabletas por sondas, en general, no se recomienda debido a la necesidad de macerar el producto, para que se diluya y se infunda por la sonda lo que puede destruir los principios activos del fármaco lo que interfiere en su actividad terapéutica . ${ }^{(15)}$

No se recomienda cambiar la estructura física de cualquier medicamento, especialmente las tabletas con recubrimiento entérico y las cápsulas de liberación prolongada. De los fármacos investigados en este estudio, en comprimidos son: fenitoína, warfarina, ciprofloxacina, gatifloxacina y moxifloxacina. La fenitoína además de presentación en comprimidos también tiene una presentación en cápsulas. .(15)

La opción de contar con cubierta entérica o no y de que la cápsula sea de liberación prolongada o no varía en función de cada laboratorio, lo que requiere que las enfermeras tengan dominio de esta información. Un comprimido tiene cubierta para su protección del pH ácido del estómago, lo que permite la liberación de la droga en el local correcto y evita la irritación gástrica (16) No deben ser triturados. En estos casos, lo ideal es solicitar al médico para reemplazar la forma sólida por líquida o inyectable. No habiendo posibilidad de estas alternativas, las presentaciones en cápsulas o comprimidos deben ser trituradas y disueltas en $30 \mathrm{ml}$ de agua, usando para lavar la sonda $30 \mathrm{ml}$ de agua estéril antes y después de la 
administración de drogas $^{(17)}$ sabiendo que al triturar se puede haber causado algún mal al fármaco.

Otro de los cuidados de enfermería relacionados con la administración de medicamentos por sonda en forma de suspensión es considerar la localización de la sonda para que pueda realizar el ajuste adecuado de la osmolaridad. El estómago apoya formulaciones de alta osmolaridad, lo que no ocurre con el intestino delgado, con el riesgo de diarrea hiperosmolar, vómitos o espasmos abdominales. Las drogas se deben diluir para lograr una osmolalidad entre 500 y $600 \mathrm{mOsm} / \mathrm{kg}^{(17)}$, teniendo en cuenta que la osmolaridad de los líquidos gastrointestinales es alrededor de 100 a 400 mOsm / kg . ${ }^{(18)}$

Otros datos relativos a la fenitoína, warfarina, ciprofloxacina, gatifloxacina y moxifloxacina es el monitoreo de las pruebas de laboratorio del paciente.

El nivel ideal terapéutico de la fenitoína es aproximadamente de 10 a $20 \mathrm{mg} / \mathrm{lmg} / \mathrm{l}$ para la mayoría de los pacientes. Los valores máximos suelen aparecer entre la cuarta y quinta hora después de la dosis, por lo que es importante que la recogida de sangre para pruebas de laboratorio se realice antes de la próxima dosis en pacientes que ya tienen un promedio de siete días de tratamiento.${ }^{(18)}$ Así, la enfermera puede ayudar a controlar las convulsiones al estar atenta al resultado de este examen.

En el tratamiento con antibióticos, se debe supervisar y evaluar, en particular, el leucograma, por el cual ocurre el recuento total de leucocitos (en adultos 4-11 millones ) así como los neutrófilos, eosinófilos, basófilos, linfocitos y monocitos permitiendo el seguimiento de la efectividad del tratamiento antibiótico. ${ }^{(18)}$ El cuadro clínico de un paciente en uso de antibióticos también debe ser observado para evaluar la evolución de síntomas tales como fiebre persistente, presencia de secreciones pulmonares y sus características, lo que puede indicar la eficacia del tratamiento. . ${ }^{(18)}$

En la terapia con warfarina, la actividad del tiempo de protrombina (PTA) es el parámetro de elección para que el enfermero supervise la acción del fármaco en el paciente. Sin embargo, para estandarizar sus resultados, el valor del tiempo de actividad de protrombina que se encuentra en la muestra del paciente se divide por el resultado de la actividad del tiempo de protrombina del plasma normal y elevado el índice de sensibilidad internacional (ISI), cuyo valor sólo varía según el lote de tromboplastina producido informado por el fabricante. El INR ideal varía de acuerdo a la terapia propuesta. Para la profilaxis y el tratamiento de la enfermedad de trombolíticos, por ejemplo, el INR ideal es de 2-3. En los pacientes con cualquier tipo de válvula cardiaca, se recomienda un rango de 2.5 a 3.5, y la recurrencia de la trombosis venosa profunda o embolia pulmonar, un INR 3-4.$^{(16)}$

Las interacciones más graves con la warfarina son aquellas que aumentan su efecto y el riesgo de sangrado, pero la disminución de su concentración plasmática y la consiguiente reducción de su efecto terapéutico, también puede dar lugar a complicaciones para el paciente. $^{(16)}$

La enfermera debe evaluar continuamente el tiempo de protrombina de la siguiente manera: en la primera semana, cada dos días o diariamente, y durante la segunda semana cada tres días. Al llegar a la dosis de mantenimiento, hacer la medición mensual. Idealmente debe mantener el tiempo de protrombina en un $25 \% .{ }^{(19)}$

Otra sustancia que debe tener monitoreo es la concentración plasmática de albúmina. Sus niveles en el adulto normal es de entre 3,6 a $5 \mathrm{~g} / \mathrm{dl}$. . ${ }^{(19)}$ 
La albúmina es una proteína que está presente en mayores cantidades en el plasma sanguíneo y es por donde las medicaciones se juntan en la sangre. Una fracción libre del fármaco administrado es capaz de superar la vascularización y ejercen su efecto farmacológico deseado en el cuerpo, pero otra parte de estos permanecen en la sangre, lo que requiere su fijación a la albúmina para que haya un aumento en la concentración sérica de la droga y, en consecuencia, aumento del riesgo de efectos adversos tales como la intoxicación. ${ }^{(19)}$

La warfarina y la fenitoína se unen a proteínas en un porcentaje del 99,9\% y $90 \%$ respectivamente, y pacientes con hipoalbuminemia pueden tener altas tasas de estos fármacos en la sangre lo que conduce al riesgo de sangrado en el caso de la warfarina, y toxicidad en caso de la fenitoína . ${ }^{(14)}$ Por último, con la intención de reducir la posibilidad de las interacciones fármaco-nutriente se debe lavar la sonda antes y después de la administración del consumo de fármacos sacando los residuos de la nutrición y medicamentos, previendo las interacciones farmacológicas y físicas como la formación de granulos y gel que pueden obstruir la sonda.$^{(14)}$ y exponre al paciente a tener que pasar ota sonda así como retarso en su nutrición.

En relación al volumen utilizado se recomienda el uso de 15 a $30 \mathrm{ml}$ de agua, dejando a la enfermera la moderación de este volumen en los pacientes que están bajo restricción hídrica. Antes y después de la irrigación de la sonda, la dieta debe ser detenida para que puedan administrarse los medicamentos. Se sugiere que esta interrupción sea de 30 a 60 minutos para todos los medicamentos administrados donde hay riesgo de interacción con la alimentación enteral. .(14)

\section{CONCLUSIÓN}

La alta incidencia de pacientes hospitalizados que utilizan cualquier tipo de soporte nutricional por sonda, así como, el uso de drogas por esta vía, aumentan la probabilidad de interacciones entre ellos.

El grupo farmacológico que mostró una mayor interacción con la nutrición enteral fue el de los antiepilépticos, especialmente la fenitoína. Todos los estudios que informaron interacción de la nutrición enteral con antibióticos llegaron a la conclusión de que no hay problema en la administración de ellos juntos, pero se considera que uno de estos estudios fue realizado en individuos sanos. Otros estudios aunque encontraron algunos cambios, no se consideraron estadísticamente significativos. La warfarina también se presentó con riesgo de interacción con la nutrición enteral.

La interacción entre el fármaco y la alimentación enteral tiene un impacto en la atención de enfermería. Las partículas del precipitado pueden provocar la obstrucción de las sondas lo que a menudo requiere el cambio de las mismas. También puede haber degradación de la droga lo que resulta en una terapia ineficaz. Así, cuando hay interacción hay también costos directos e indirectos. Es necesario cambiar la sonda, detener la alimentación enteral, administrar otra dosis del medicamento, causando retrasos en la terapia y trabajo extra del personal de enfermería, todas consecuencias negativas para la calidad de los cuidados de enfermería, y molestias a los pacientes, pues a menudo pasar una sonda es un proceso traumático y doloroso.

Como las enfermeras tienen la responsabilidad del proceso de programación, preparación y administración de medicamentos, y paralelo a ello, también participan en el proceso de la terapia nutricional es necesario conocer acerca de las acciones nutricionales, farmacológicas 
y las posibles interacciones de manera que ambas terapias no se vean perjudicadas y hasta suprimir una a expensas de la otra, con el fin de garantizar un tratamiento eficaz al que el paciente tiene derecho.

La administración de los medicamentos y la seguridad nutricional representa un reto para el equipo de enfermería, teniendo en cuenta que son los profesionales directamente implicados en la preparación y administración de ambas terapias. Hay que realizar estudios experimentales utilizando mezclas de fármacos con nutrición enteral con la finalidad de comprender mejor el margen de seguridad de la atención de enfermería en medicina, para garantizar la eficacia de la terapia enteral y la farmacología.

\section{REFERENCIAS}

1. Agencia Nacional de Vigilância Sanitária. ANVISA. [Citado 2008 mar 18].Disponível em: http://www.anvisa.gov.br/medicamentos/conceito.htm\#3.2 .

2-Jiménez NVT, Merino MS, Ordovás JPB, Casabó VGA. Interacciones entre medicamentos V alimentos: bases farmacoterapéuticas. Valencia: Convaser. Espana. 1999.

3. Cintra EA, Nishide VM, Nunes WA. Assistência de enfermagem ao paciente gravemente enfermo.2 $2^{\mathrm{a}}$ ed. São Paulo: Atheneu; 2005.

4.Peterlini MAS,Chaud MN,Pedreira MLG. Incompatibilidade entre fármacos e materiais de cateteres e acessórios da terapia intravenosa[Online]2006; 5(3):234-41.[Acesso 2008 jun 24]. Disponível em : http://www.uff.br/objnursing/index.php/nursing/article/view/483/109.

5. Clayton BD, Stock YN. Farmacologia na prática de enfermagem. 13aㅡ ed. Rio de Janeiro: Elsevier; 2006.

6. Polit DF, Beck CT, Hungler BP. Fundamentos de Pesquisa em Enfermagem. 5a ed. Porto Alegre: ArtMed; 2004.

7.Faraji B, Yu PP. Serum phenytoin levels of patients on gastrostomy tube feeding. Journal of Neuroscience Nursing[Online] 1998; 30 (1): 55-9. [Acesso em 2008 jul 24]. Disponível em: http://findarticles.com/p/articles/mi hb6374/is /ai n28705407?tag=artBody;col1

8. Sanz HM, González EHP, Alcaraz MJT, Luque RI, Requena TC. Seguimiento de la administración de medicamentos por sonda nasogástrica: elaboración de una guía práctica. Nutrición hospitalaria,2000;15(6):291-301.

9. Cerulli J, Malone M. Assement of drug-related problems in clinical nutrition patients. J. Parenter Enteral Nut. 1999; 23(4): 218-221.

10. Burkhardt O, Satss H, Thus U, Bormer K, Welte T. Effects of enteral feeding on the oral bioavailability of moxifloxacin in healthy volunteers. C. Pharmacokinetics 2005; 44 (9): 969 76.

11. Mimoz O, Binter V, Jacolot A, Edouard A, Tod M, Petitjean O, Samii K. Pharmacokinetics and absolute bioavailability of ciptofloxacin administered through a nasogastric tube with contiunuous enteral feeding to critically ill patients. J.Intensive Care Medicine 1998; 24(10): 1047-51.

12. Kanji S, Mckinnon PS, Barletta JF, Kruse JA, Devlin JW. Bioavailability of gatifloxacin by gastric tube administration with and without concomitant enteral feeding in critically ill patients. Critical Care Medicine 2003; 31 (5): 1347-52.

13. Dickerson RN, Garmon WN, Kuhl DA, Minard G, Brown RO. Vitamin K-independent warfarin resistance after concurrent administration of warfarin and continuous enteral nutrition. Pharmacotherapy 2008; 28 (3): 308-13.

14. Silva LD, Pereira SM, Freitas AM. Assistência ao paciente crítico. 2ª̄ed.Rio de Janeiro:Cultura Medica, 2005.

15. Mahan LK, Stump SE. Alimentos, Nutrição e Dietoterapia. 11a ed. São Paulo: Roca; 2005.

16. Katzung BG. Farmacologia Básica e Clinica. 10ª ed. São Paulo: Mc Graw-Hill; 2007. 
17.Gilbar PJ. A guide to enteral drug administration in palliative care. J. pain symptom management 1999; 17 (3): 197-207.

18. Nuhoeq C. Administración de medicamentos em pacientes com nutrición enteral mediante sonda. Nutrición Hospitalaria 2006; 21 (supl. 4): 199 - 216.

19. Lechuga MG, Estela AC, Pera DC, Riba RF, Cels IC, Falgás JB. Importância de lãs características físico-químicas de los fármacos para su administración por sonda nasoentérica o enterostomía. Barcelona: Farmacia Hospitalaria. 1998; 22 (3): 1-7. 